\title{
Nonlinearity in holey optical fibers: measurement and future
} opportunities

\author{
N. G. R. Broderick, T. M. Monro, P. J. Bennett and D. J. Richardson \\ Optoelectronics Research Centre, University of Southampton, \\ Southampton, SO17 1BJ, UK. \\ Phone: $+44(0) 2380593144$, Fax: $+44(0) 2380593142$ \\ email: ngb@orc.soton.ac.uk
}

June 9, 1999

\begin{abstract}
Holey fibers combine two dimensional microstructuring with one dimensional longitudinal propagation to produce fibers with tailorable dispersive and nonlinear properties. In this paper we measure the effective nonlinearity of a typical holey fiber. The small effective area possible in this type of fiber significantly enhances their effective nonlinearity relative to standard fiber.
\end{abstract}

Holey fibers (HF) provide a new paradigm for the transverse guidance of light. Unlike conventional optical fibers, which use different core and cladding materials, HFs can be made from a single material, and guidance is provided by the average index difference between the core and cladding $[1,2]$. This difference arises from holes in the cladding, which can either be randomly or periodically arranged. When the holes are periodically arranged such structures can also possess a complete photonic bandgap[3]. The HF characterized here is shown in Fig. 1, and although this fiber guides by average index effects, the techniques described here can also be applied to photonic bandgap fibers.

Holey fibers are attractive for photonic devices because their optical properties can be engineered during fabrication. They can have anomalous waveguide dispersion and be rigorously single-mode, which is impossible in conventional step-index fibers[4]. The HF from Fig. 1 is predicted to always have anomalous total dispersion and furthermore is single moded over a wide wavelength range[5], -truly unique properties for an optical fiber. Such fibers offer the potential for creating soliton fiber lasers for $\lambda<1.3 \mu \mathrm{m}$. In 
particular, doping a HF with ytterbium would allow easy fabrication of sub-picosecond high repetition pulse sources at $1 \mu \mathrm{m}$ which would find use in a variety of areas.

Soliton formation relies on a balance between the fiber dispersion and nonlinearity. It is then natural to ask how the holes affect the effective nonlinearity of $\mathrm{HF}$, and whether or not it is possible to significantly alter it via the fiber design. Holey fibers offer two distinct ways to achieve this. Firstly, by appropriate choice of HF geometry, the mode size can be tailored by as much as three orders of magnitude[6], a much larger range than that possible in conventional fiber types. Changing the mode size alters the effective nonlinearity of the fiber by increasing/decreasing the intensity inside the fiber which increases/decreases the nonlinear phase change experienced by light during propagation. A more direct way to effect the nonlinearity would be by using HFs in which the hole spacing is less than the wavelength of the light. In this regime a large fraction of the mode can propagate in the air[7], and thus by filling the HFs with a suitable nonlinear material the effective nonlinearity could be significantly enhanced.

To measure the nonlinearity of our fiber we used the method of Boskovic et al. [8] which involves the use of high power dual frequency beat signals. The fiber nonlinearity creates spectral sidebands (see Fig. 2) and the intensity ratio between the signal and the first side band gives the nonlinear phase through

$$
\frac{I_{0}}{I_{1}}=\frac{J_{0}^{2}\left(\phi_{S P M} / 2\right)+J_{1}^{2}\left(\phi_{S P M} / 2\right)}{J_{1}^{2}\left(\phi_{S P M} / 2\right)+J_{2}^{2}\left(\phi_{S P M} / 2\right)}
$$

where $I_{0,1}$ are the peak intensities of the signal and first sideband respectively, $J_{n}$ is the nth Bessel function. $\phi_{S P M}$ is the nonlinear phase shift given by

$$
\phi_{S P M}=\frac{2 \omega_{0}}{c} \gamma L P
$$

where $L$ is the effective fiber length and $P$ the signal power. Using this method, the effective nonlinearity. $\gamma$ can be calculated for the fiber under test. Note that

$$
\gamma=\frac{n_{2}}{A_{e f f}}
$$

where $A_{e f f}$ is the effective area, as defined in Ref. [9], and $n_{2}$ depends on the material composition of the fiber. The HFs considered here (for example Fig. 1) are composed solely of silica, and so $n_{2}$ is known. Hence this method provides a direct way of accurately measuring the effective mode area $A_{\text {eff }}$ in HF.

This technique for measuring the effective area is particularly useful for HFs, as it makes no assumptions about the mode shape. As can be seen from Fig. 1, the mode 
of this HF has 6-fold (hexagonal) symmetry, and cannot be approximated by a simple circularly-symmetric Gaussian. This is particularly noticeable in the wings or at shorter wavelengths, where the mode becomes more hexagonal. More traditional methods, which rely on Gaussian optics to estimate the area, would fail in these cases.

To perform the nonlinear measurements we used a diode-seeded erbium doped amplifier chain. The input to the chain was derived from two tuneable DFB lasers which were coupled together and the resultant beat signal externally modulated to produce 5 ns square pulses at a repetition rate of $200 \mathrm{kHz}$. After amplification the peak power in the pulses was $\approx 100 \mathrm{~W}$, and we coupled roughly $50 \%$ of this power into the HF (length $1.175 \mathrm{~m}$ ). We also tested $(1.9 \mathrm{~m})$ of dispersion shifted fiber (DST) to test the procedure. As these experiments use relatively short lengths of fiber fiber dispersion can be neglected.

Fig. 2 shows the incident and transmitted pulse spectra for high incident power onto the HF. Both the signal beams can be clearly seen and are separated by $0.23 \mathrm{~nm}$ and hence the nonlinearly generated side bands are also separated by the same amount from the signal beam. From the input spectrum (dashed line) it can be seen that the amplification process is essentially linear although a small nonlinear phase shift is observed (note the additional low level side bands). The level of the first order sidebands is dramatically increased after propagation through the fiber, and in addition further sidebands are generated.

We recorded the output spectra at a range of powers and measured the ratio of $I_{0} / I_{1}$ as a function of transmitted power. The total nonlinear phase at each power level was obtained by taking the inverse of Eq. 1. The nonlinear phase shift due to propagation in the fiber under test was then evaluated by subtracting the small nonlinear phase shift associated with propagation through the amplifier chain. The results obtained for both the DSF and HF are shown in Fig. 3 along with the results of least squares linear fits.

As expected the nonlinear phase increases linearly with peak power and the slope of the fit gives the nonlinearity via Eq. 2 . For the DSF, the value $\gamma \approx 4.32 \times 10^{-10} \mathrm{~W}^{-1}$ was obtained, which using the standard value of $n_{2}=2.35 \times 10^{-20} \mathrm{~m}^{2} \mathrm{~W}^{-1}$ for DSF gives a mode area of $A_{\text {eff }} \approx 54 \mu \mathrm{m}^{2}$ (see Eq. 3), a realistic value for this fiber type $[8,9]$. For the holey fiber we obtained $\gamma \approx 1.56 \times 10^{-10} \mathrm{~W}^{-1}$. Note that we have included a correction of $\approx 5 \%$ to account for the effect of the background loss of $0.24 \mathrm{dBm}^{-1}$ in the $\mathrm{HF}$ [5]. As the HF is formed from pure silica, $n_{2}=2.16 \times 10^{-20} \mathrm{~m}^{2} \mathrm{~W}^{-1}$ [9], and so for this fiber $A_{\text {eff }} \approx 13.9 \mu \mathrm{m}^{2}$. Applying our numerical model for HFs [6], we predict that $A_{\text {eff }} \approx 14 \mu \mathrm{m}^{2}$, in excellent agreement with the above measurement.

From this excellent agreement and from Fig. 1, it is clear that very little light is located 
in the holes. However by careful fiber design, it is possible to locate as much as $40 \%$ of the mode within the holes [7]. In such a case the effective area is no longer a useful measure of the nonlinearity, as different parts of the mode see radically different nonlinearities, and the only meaningful parameter would be $\gamma$, which is what is measured by this method.

To determine whether the microstructuring in $\mathrm{HF}$ effects the Brillouin scattering, we measured the relative Brillouin thresholds for the HF and DSF. We used the experimental setup described above, but amplified the modulated output from a single DFB laser. The pulse duration and source linewidth were $10 \mathrm{~ns}$ and $\$ 300 \mathrm{MHz}$ respectively. By coupling the reflected light into a high resolution $(0.02 \mathrm{~nm})$ commercial spectrum analyzer we were able to resolve the Brillouin line and directly measure the threshold. For this measurement we ensured that the lengths of HF and DSF were the same $(1.175 \mathrm{~m})$, and we decreased the repetition rate to $5 \mathrm{kHz}$. The resulting peak power of $4 \mathrm{~kW}$ was sufficient to observe the generation of multiple Brillouin lines in both fibers (see Fig. 4). For the HF the threshold was $75 \mathrm{~W}$ whereas for the DSF it was $275 \mathrm{~W}$. The ratio of these thresholds is 3.66 which agrees within experimental error with the ratio of the effective areas $(54 / 13.9 \approx 3.88)$ and thus we conclude that in this HF there is no significant change to the phonon mode densities due to the microstructured cladding region. The Brillouin shift for the HF was measured to be $11.5( \pm 0.5) \mathrm{GHz}$, consistent with the quoted value for pure silica [9].

In conclusion, we have successfully measured the Kerr nonlinearity and the Brillouin threshold for a typical holey fiber, and we find that both are enhanced by the small effective area of this HF $\left(\approx 14 \mu \mathrm{m}^{2}\right)$. Note that mode areas as small as $1 \mu \mathrm{m}^{2}$ are possible for different hole arrangements. The technique described here is a fast easy way to measure the effective area of HFs since $n_{2}$ in such fibers is that of pure silica. The measured effective area is in excellent agreement with the theoretical prediction, providing additional support for our model of $\mathrm{HFs}[6]$.

This particular fiber, which is predicted to be anomalously dispersive at all wavelengths [5], could thus be used in a laser cavity to provide soliton mode-locking over a wide wavelength range. Our model predicts that the dispersion of this fiber at $1.03 \mu \mathrm{m}$ is $\approx 20 \mathrm{ps} \mathrm{nm}^{-1} \mathrm{~km}^{-1}$, similar to that of standard fiber at $1.55 \mu \mathrm{m}$. Hence, for example, when a 1 ps input pulse is used, the soliton period is $140 \mathrm{~m}$, similar to that of standard fiber at $1.55 \mu \mathrm{m}$. Also, the effective nonlinearity for this $\mathrm{HF}$ is 3.9 times that of standard fiber due to the smaller effective area, and thus soliton energies would be reduced by this factor. This increase in the effective nonlinearity also reduces the threshold for other nonlinear effects such as continuum generation[10] and four wave mixing. 


\section{References}

[1] J.C. Knight, B. Managan, T.A. Birks, P.St.J. Russell, and J.P. de Sandro, Opt. Lett. 21, 1547 (1996).

[2] T.A. Birks, J.C. Knight, and P.St.J. Russell, Opt. Lett. 22, 961 (1997).

[3] J.C. Knight, J. Broeng, T.A. Birks, and P.St.J. Russell, Science 282, 1476 (1998).

[4] J.M. Senior, Optical Fiber Communications (Prentice Hall International Ltd, Maylands Avenue, Hempstead, UK, 1992).

[5] P.J. Bennett, T.M. Monro, and D.J. Richardson; 'Fabrication, Splicing and Characterization of Practical Holey Fibres', European Conference on Communications (accepted) (1999).

[6] T.M. Monro, D.J. Richardson, N.G.R. Broderick, and P.J. Bennett, J. Light. Tech. 17, (1999).

[7] T.M. Monro, D.J. Richardson and P.J. Bennett, Conference on Lasers and ElectroOptics, OSA Technical Digest (Optical Society of America, Washington DC, 1999), pp 158.

[8] A. Boskovic, S.V. Chernikov, J.R. Taylor, L. Gruner-Nielsen, and O.A. Levring, Opt. Lett. 21, 1966 (1996).

[9] G.P. Agrawal, Nonlinear Fibre Optics (Academic Press, San Diego, 1989).

[10] J.K. Ranka, R.S. Windeler, and A.J. Stentz, Conference on Lasers and Electro-Optics, OSA Postdeadline Technical Digest (Optical Society of America, Washington DC, 1999), CPD8. 
Figure 1: SEM of the holey fiber used here along with the predicted mode profile at $1550 \mathrm{~nm}$, with contours separated by $1 \mathrm{~dB}$. Note that the mode is not circular.

Figure 2: Typical measured spectrum for the nonlinear mixing in the holey fiber. The dashed line is the input spectrum, the solid line is the transmitted spectrum. Note the large increase in power in the sidebands due to SPM.

Figure 3: Experimental plot of the nonlinear phase against internal peak power for both the HF (squares) and the DSF (dots). The least squares fits to the experimental data are shown.

Figure 4: Spectra of the Brillouin spectra for HF (solid line) and DSF (dashed line) for the same transmitted power. Note that multiple Brillouin lines are generated in the HF due to the smaller effective area. 


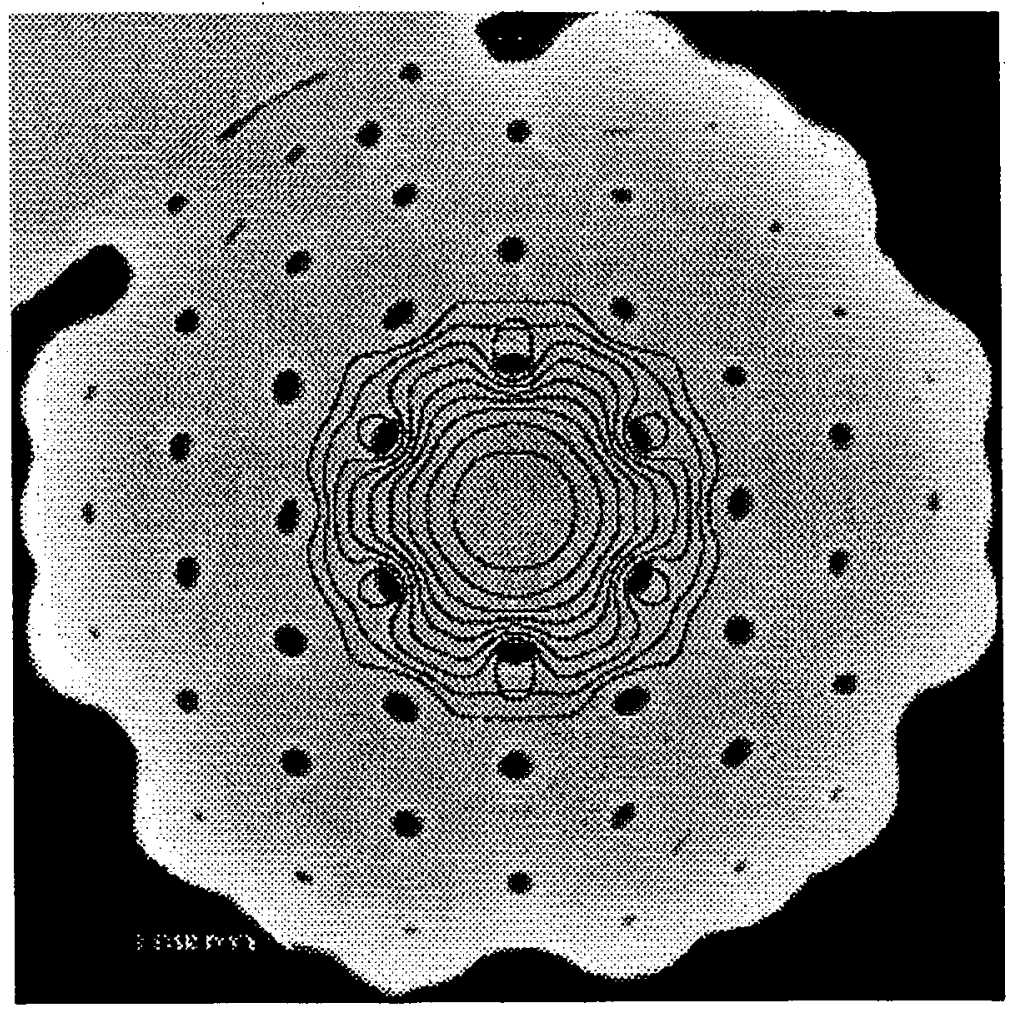

Fig. 1 Broderick et al., Optics Letters 


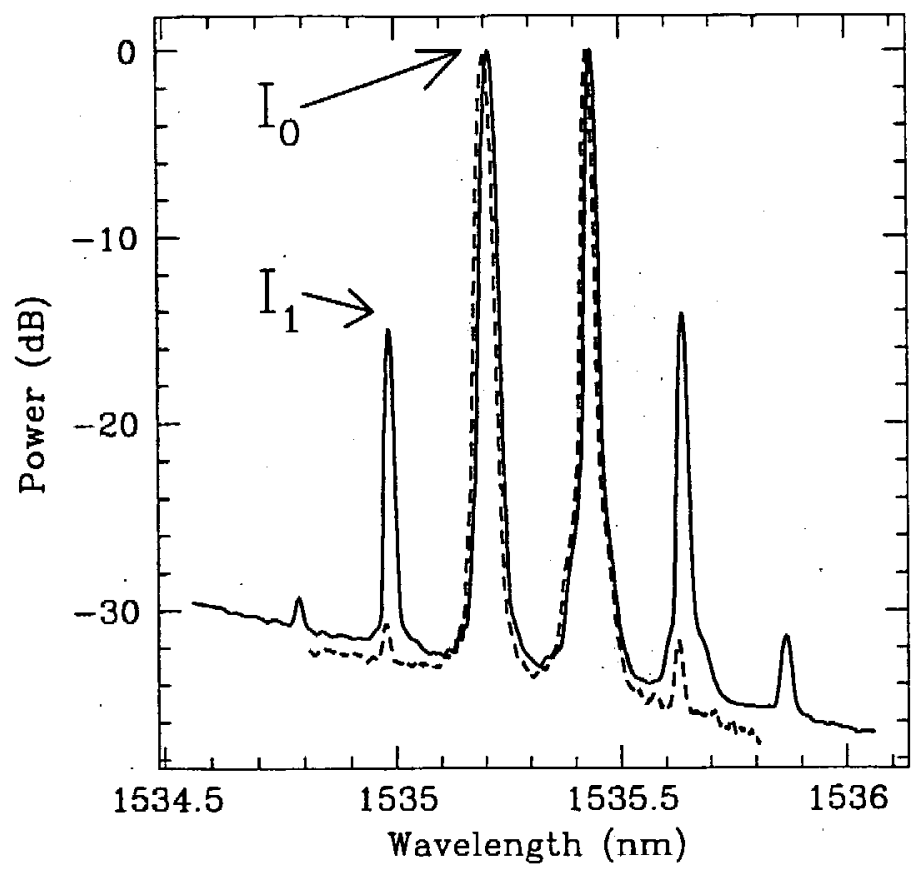

Fig. 2 Broderick et al., Optics Letters 


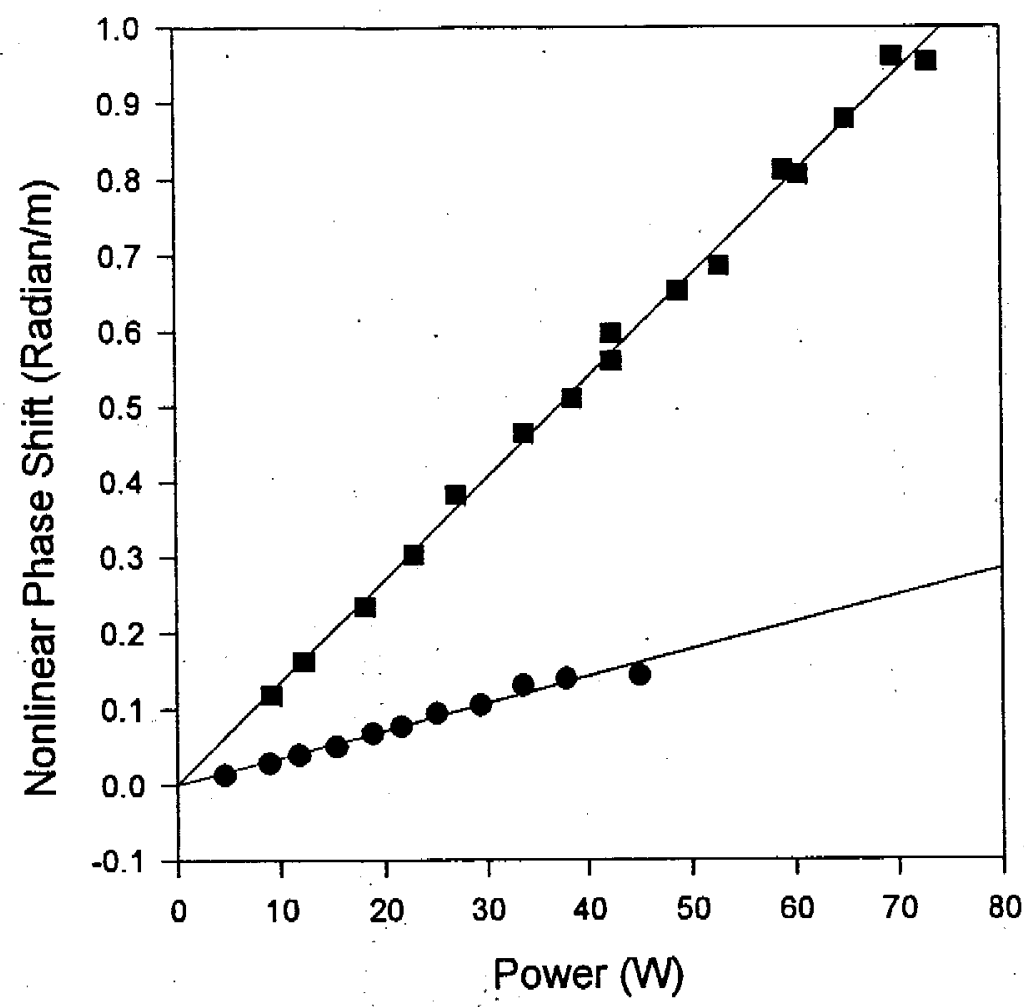

Fig. 3 Broderick et al., Optics Letters 


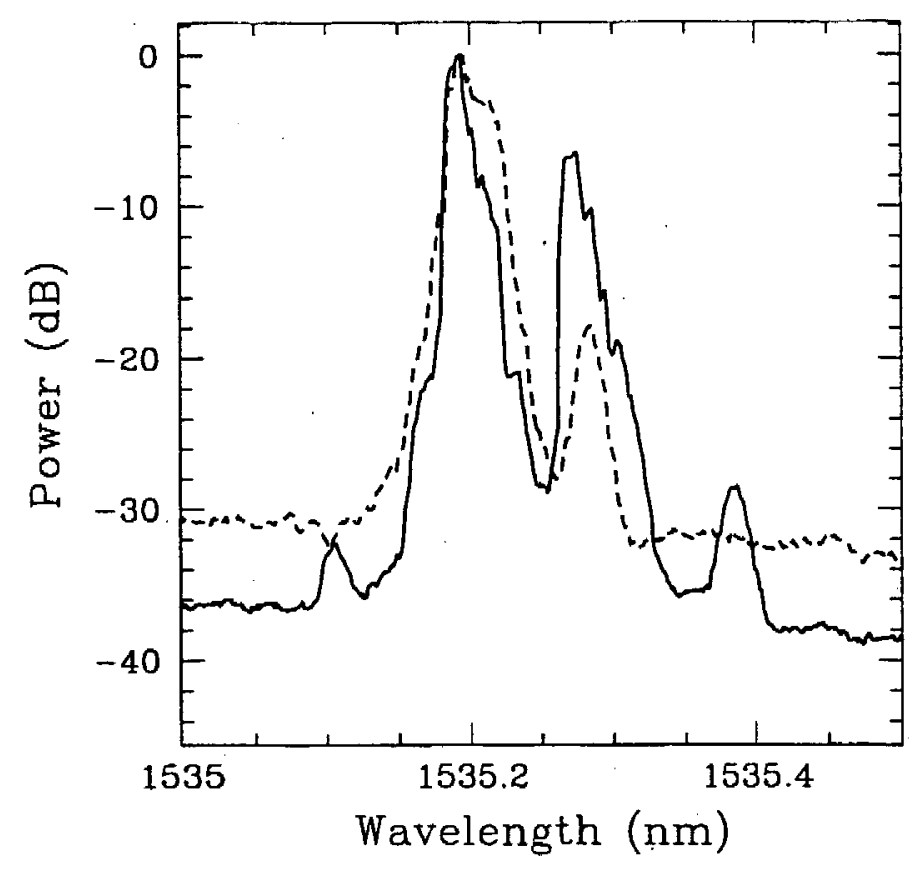

Fig. $4 \quad$ Broderick et al., Optics Letters 